ACCEPTED For PUblicAtion In ApJ

Preprint typeset using LTEX style emulateapj v. 08/22/09

\title{
THE ULTRALUMINOUS X-RAY SOURCES NEAR THE CENTER OF M82
}

\author{
A. K. H. KONG ${ }^{1}$, Y. J. YANG ${ }^{2}$, P.-Y. HSIEH ${ }^{2}$, D. S. Y. MAK ${ }^{3}$, AND C. S. J. PUN ${ }^{3}$ \\ Accepted for publication in ApJ
}

\begin{abstract}
We report the identification of a recurrent ultraluminous X-ray source (ULX), a highly absorbed X-ray source (possibly a background AGN), and a young supernova remnant near the center of the starburst galaxy M82. From a series of Chandra observations taken from 1999 to 2005, we found that the transient ULX first appeared in 1999 October. The source turned off in 2000 January, but later reappeared and has been active since then. The X-ray luminosity of this source varies from below the detection level $\left(\sim 2.5 \times 10^{38} \mathrm{ergs} \mathrm{s}^{-1}\right)$ to its active state in between $\sim 7 \times 10^{39} \mathrm{ergs} \mathrm{s}^{-1}$ and $1.3 \times 10^{40} \mathrm{ergs} \mathrm{s}^{-1}$ (in the $0.5-10 \mathrm{keV}$ energy band) and shows unusual spectral changes. The X-ray spectra of some Chandra observations are best fitted with an absorbed powerlaw model with photon index ranging from 1.3 to 1.7. These spectra are similar to those of Galactic black hole binary candidates seen in the low/hard state except that a very hard spectrum was seen in one of the observations. By comparing with near infrared images taken with the Hubble Space Telescope, the ULX is found to be located within a young star cluster. Radio imaging indicates that it is associated with a H II region. We suggest that the ULX is likely to be a $>100 M_{\odot}$ intermediate-mass black hole in the low/hard state. In addition to the transient ULX, we also found a highly absorbed hard X-ray source which is likely to be an AGN and an ultraluminous X-ray emitting young supernova remnant which may be related to a 100-year old gamma-ray burst event, within 2 arcsec of the transient ULX.

Subject headings: black hole physics — galaxies: individual (M82) — supernova remnants — X-rays: binaries - X-rays: galaxies
\end{abstract}

\section{INTRODUCTION}

Ultraluminous X-ray sources (ULXs) are defined as offnuclear X-ray sources with isotropic luminosities much higher than the Eddington limit for a solar mass black hole $\left(L_{X} \sim 1.3 \times 10^{38} \mathrm{ergs} \mathrm{s}^{-1}\right)$. Typical X-ray luminosities of ULXs are in between $10^{39} \mathrm{ergs} \mathrm{s}^{-1}$ and $10^{41} \mathrm{ergs} \mathrm{s}^{-1}$. The physical nature of ULXs has been an enigma because of their high energy output. Many ULXs show strong variability suggesting that they are accreting compact objects. Assuming the emission is isotropic, then some of the ULXs may harbor intermediate-mass black hole (IMBH; Colbert \& Mushotzky 1999; Makishima et al. 2000) with masses of $100-10000 M_{\odot}$. Alternatively, ULXs may simply be stellarmass black holes. It has been suggested that ULXs are stellarmass black holes with radiation pressure-dominated (Begelman 2002) or slim (Ebisawa et al. 2003) accretion disks that cause super-Eddington luminosities. Furthermore, ULXs may be stellar-mass black holes with anisotropic X-ray emission (King et al. 2001), or micro-blazars which happened to be observed along the direction of their relativistically beamed jet (Körding et al. 2002). In addition, some ULXs may be young X-ray luminous supernova remnants in a highdensity medium, or hypernova remnants. Finally, some ULXs have been identified with background AGNs through optical follow-up spectroscopy (e.g., Foschini et al. 2002; Masetti et al. 2003). Each of these models has difficulties to fully explain the observations, but yet has some supporting pieces of evidence. Currently, we do not have a complete picture about the physical nature of ULXs, primarily because we do not

\footnotetext{
${ }^{1}$ Kavli Institute for Astrophysics and Space Research, Massachusetts Institute of Technology, Cambridge, MA 02139

${ }^{2}$ Institute of Astronomy and Astrophysics, Academia Sinica, Taipei, Taiwan

${ }^{3}$ Department of Physics, University of Hong Kong, Pokfulam, Hong Kong
}

have dynamical mass measurements of the compact objects that power ULXs.

In our current stellar formation and evolution theory, we are only able to constrain two classes of black holes, the supermassive black holes with masses exceeding $10^{6} M_{\odot}$ at the center of galaxies and stellar-mass black holes with masses lower than $20 M_{\odot}$. If some ULXs host IMBHs, they might provide a clue to fill in the missing link between stellar-mass black holes and supermassive black holes.

In this paper, we report on a recurrent transient ULX in the starburst galaxy M82 by using archival Chandra and $\mathrm{Hub}$ ble Space Telescope (HST) data. The source is located near the galactic dynamical center and is one of the most luminous X-ray sources within the supper-bubble region of M82 (Matsushita et al. 2005; Matsumoto et al. 2000), close to several super-star clusters. This source is probably the second most luminous source in M82. The most luminous source, $\mathrm{M} 82 \mathrm{X}-1$, is a prime candidate of IMBH and is about 5 arcsec from the transient (see Kaaret et al. 2006 and references therein). In addition, we also study the physical nature of the two ULXs very close to the ultraluminous transient by using multi-wavelength data.

In $\S 2$ we describe the Chandra and HST observations. We present the data analysis and results in $\S 3$. A discussion of the three ULXs is given in $\S 4$.

\section{OBSERVATIONS}

\subsection{Chandra}

M82 (NGC 3034) is a nearby starburst galaxy. We adopt a distance of 3.6 Mpc to M82 based on the Cepheid distance of $3.63 \pm 0.34 \mathrm{Mpc}$ to its close neighbor galaxy M81 (Freedman et al. 1994). M82 was observed twelve times between the year of 1999 and 2005 with Chandra. The details of the observations are given in Table 1. Among these twelve observations, observations 3 and 5 were using the High Resolution Camera 
TABLE 1

Chandra OBSERVATION LOG

\begin{tabular}{clcccc}
\hline \hline Index & Date & ObsId & Exposure & Instrument & Remark \\
\hline 1 & $1999-09-20$ & 361 & $33.7 \mathrm{ks}$ & ACIS-I & \\
2 & $1999-09-20$ & 1302 & $15.7 \mathrm{ks}$ & ACIS-I & \\
3 & $1999-10-28$ & $1411-1$ & $36.3 \mathrm{ks}$ & HRC-I & \\
4 & $1999-12-30$ & 378 & $4.2 \mathrm{ks}$ & ACIS-I & off-axis \\
5 & $2000-01-20$ & $1411-2$ & $17.8 \mathrm{ks}$ & HRC-I & \\
6 & $2000-03-11$ & 379 & $9.1 \mathrm{ks}$ & ACIS-I & off-axis \\
7 & $2000-05-07$ & $380-1$ & $3.9 \mathrm{ks}$ & ACIS-I & off-axis \\
8 & $2000-06-12$ & $380-2$ & $1.2 \mathrm{ks}$ & ACIS-I & off-axis \\
9 & $2002-06-18$ & 2933 & $18.3 \mathrm{ks}$ & ACIS-S & \\
10 & $2005-02-04$ & 6097 & $58.2 \mathrm{ks}$ & ACIS-S & off-axis, $1 / 8$ subarray \\
11 & $2005-08-17$ & 5644 & $75.1 \mathrm{ks}$ & ACIS-S & $1 / 8$ subarray \\
12 & $2005-08-18$ & 6361 & $19.2 \mathrm{ks}$ & ACIS-S & $1 / 8$ subarray \\
\hline
\end{tabular}

NOTE. - ObsIDs 1411 and 380 have two observations merged in one event list. We used a time filter to separate the two observations.

(HRC-I). The rest were using the Advanced CCD Imaging Spectrometer array (ACIS-I or ACIS-S). We used CIAO v3.3, HEAsoft v6.2, and XSPEC v11.3 packages to perform data reduction and analysis.

For ObsIDs 1411 and 380, there are two separate observations within the same event list. Therefore, we used a timefilter to split the observations and analyzed the data separately (observations 3, 5, 7, and 8). Five of the observations (4, 6, 7, 8 , and 9) are off-axis to reduce pile-up of M82 X-1 due to its high luminosity. In particular, during observations $10-12$, the detector employed a $1 / 8$ subarray mode with a frame time of 0.441 s to reduce pile-up.

\section{2. $H S T$}

Since M82 has high extinction near its core where our targets are located, we used near IR image to study the environment around the X-ray sources. We obtained HST Near Infrared Camera and Multi-Object Spectrometer (NICMOS) data from the Multimission Archive at STScI (MAST). M82 was observed on 1998 April 11 by using the NIC2 camera with the F160W filter ( $H$-band). The NIC2 camera has a resolution of $0^{\prime \prime} .075 \mathrm{pixel}^{-1}$ and a field-of-view of $19.2 \times 19.2$ $\operatorname{arcsec}^{2}$. To cover the central 1.5 arcmin along the semimajor axis, we obtained 11 pipeline processed NICMOS images from the MAST. The individual images were combined to form a mosaic that were used for analysis. To correct the absolute astrometry of the NICMOS image, we aligned the NICMOS image with a wide-field HST ACS mosaic image (Mutchler et al. 2005). The ACS mosaic has a dimension of $10^{\prime} .24 \times 10^{\prime} .24$ and can be obtained as High-Level Science Products via the MAST ${ }^{4}$. The observations and data reduction are described in Mutchler et al. (2005). We used the F814W ( $I$ band) mosaic for our analysis and corrected the absolute astrometry by using the $2 \mathrm{MASS}$ catalog. We identified 21 isolated stars in the field and matched with the 2MASS catalog. Using IRAF tool ccmap, we corrected the absolute astrometry of the ACS mosaic with a registration error of 0.2 arcsec. We then registered the NICMOS mosaic with the astrometric corrected wide-field ACS image. With 9 isolated stars in both field-of-views of NICMOS and ACS mosaics, we corrected the astrometry of the NICMOS image with a registration error of 0.036 arcsec.

\section{DATA ANALYSIS AND RESULTS}

\subsection{X-ray Imaging}

\footnotetext{
${ }^{4}$ http://archive.stsci.edu/prepds/m82
}

The brightest source in the field of M82 is M82 X-1 (CXOU J095550.2+694047; see Fig. 1). We note that the X-ray coordinates are based on an astrometric corrected image (see §3.5). About 5 arcsec southeast of M82 X1 , there is a complex of three bright X-ray sources (Matsumoto et al. 2001). By examining the Chandra images, the brightest one, CXOU J095551.0+694045 (J095551.0 hereafter; note that in Matsumoto et al. 2001, the source is called J095551.1+694045 due to different astrometry), was clearly below the detection limit in two observations indicating that it is a highly variable source. Note that previous studies have mentioned the transient behavior of this source (Matsumoto et al. 2001; Kaaret et al. 2006). In addition to J095551.0, there are several additional transients as shown in Figure 1; the discussion of these transients is out of the scope of this paper. There are two fainter X-ray sources, CXOU J095551.2+694044 (J095551.2 hereafter) and CXOU J095550.6+694044 (J095550.6 hereafter) located at about 2 arcsec to the south of J095551.0 forming a triangle (Fig. 1). In contrast to J095551.0, these two sources are always active.

\subsection{X-ray Spectroscopy}

We performed spectral analysis for all Chandra ACIS data by using XSPEC v11.3. We also used CIAO's Sherpa for independent check. In three of the observations, the X-ray sources are located near the aim point and we can use a circular extraction region with radii of $0.8-1.3$ arcsec depending on the contamination of nearby sources. The relatively small extraction radii were used because the three $\mathrm{X}$-ray sources that we are interested in are close to each other. For the remaining four observations, our targets were off-axis and therefore we used an elliptical region to extract the spectra. For the background, we used a nearby source free region. We rebinned the $0.3-7 \mathrm{keV}$ spectra with at least 20 counts per spectral bin, and used $\chi^{2}$ statistics to find the best-fitting parameters. Corresponding response files were generated using CIAO.

For the transient (J095551.0), the X-ray spectra can be adequately fitted with an absorbed power-law model. The spectral parameters are listed in Table 2 . In general, the photon index varies between 1.3 and 1.7 while the $N_{H}$ is about $3 \times 10^{22}$ $\mathrm{cm}^{-2}$, consistent with the extinction measured with near IR observations (Alonso-Herrero et al. 2003). It is worth noting that during observation 9 , the source suffers mild pile-up. In this case, we included a pile-up model in spectral fit yielding a pile-up fraction of $\sim 15 \%$. For consistency check, we also applied a pile-up model for other observations. Pile-up affects some of the observations (in particular observations 4 , 6,7 and 8) with a maximum pile-up fraction of $\sim 10 \%$. Our spectral fit of observation 10 is different comparing to Kaaret et al. (2006). This is likely due to the diffuse background and contamination of nearby sources. We used a nearby diffuse emission region as the background and the fit was performed with the background subtracted spectrum. We also used a smaller extraction region to reduce the contamination of nearby sources. In fact, Kaaret et al. (2006) required an additional very soft component to fit the spectrum indicating the contribution of diffuse emission and nearby sources. We also performed a fit with a bigger extraction region and without background subtraction. The result is consistent with Kaaret et al. (2006). Since five of the observations $(4,6,7,8$, and 10) are off-axis, the spectra of the transient are contaminated by J095551.2 and J095550.6. The contamination is particularly serious in observations $4,6,7$, and 8 , and may result the rel- 

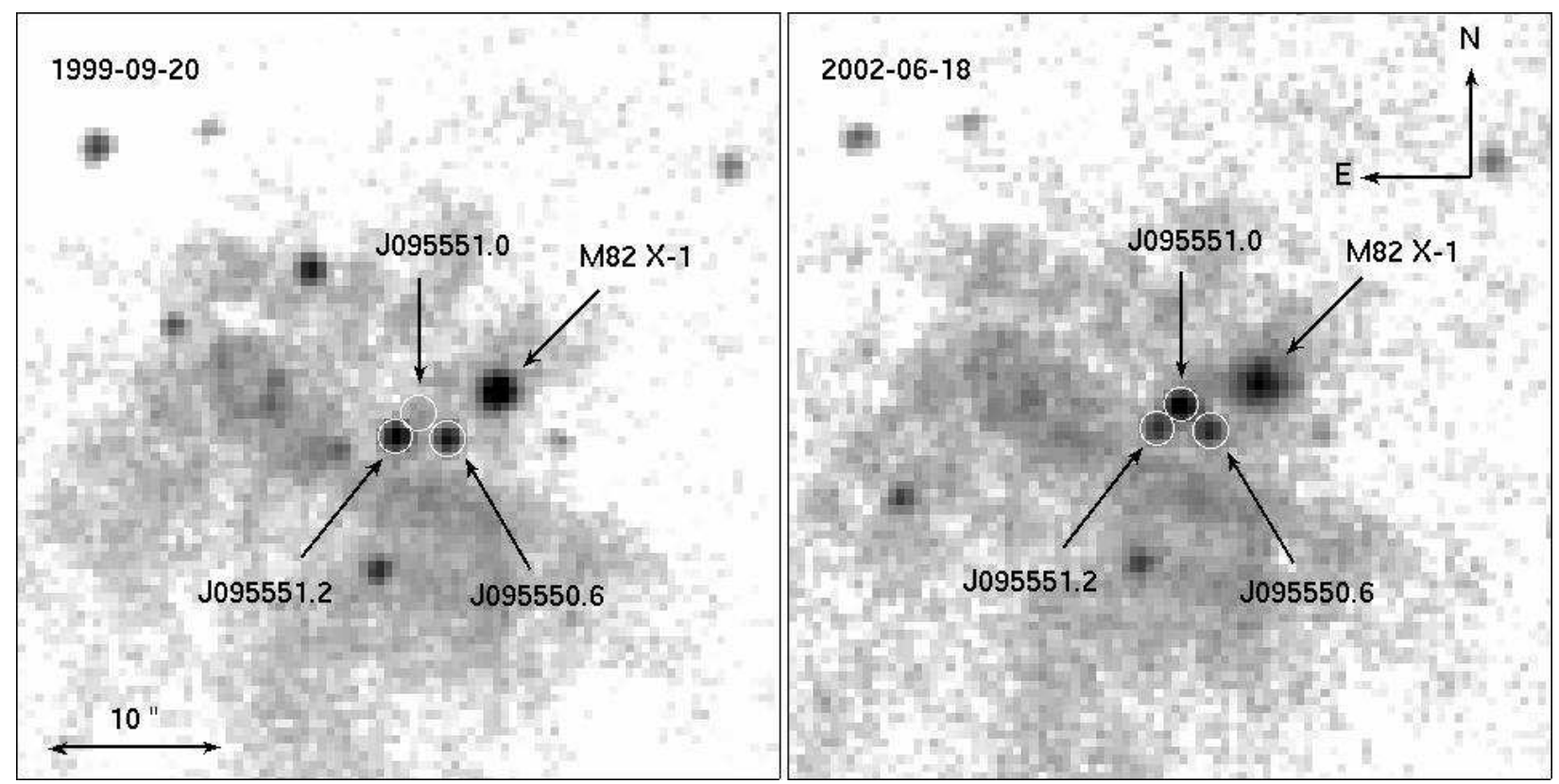

FIG. 1.- Chandra 0.3-7 keV images of the central $45^{\prime \prime} \times 45^{\prime \prime}$ region of M82 as seen on 1999 September 20 (Left; observation 1) and 2002 June 18 (Right; observation 9). Both figures have the same spatial scale. The locations of the three ULXs discussed in this paper are marked. We also indicate the position of $\mathrm{M} 82 \mathrm{X}-1$. The images has been slightly smoothed with a $0.5^{\prime \prime} \sigma$ Gaussian function.

TABLE 2

POWER-LAW SPeCTRAL MOdel FOR THE ULXs

\begin{tabular}{|c|c|c|c|c|c|c|c|c|c|c|c|c|}
\hline \multirow[t]{2}{*}{ Observation } & \multicolumn{4}{|c|}{ J095551.0 } & \multicolumn{4}{|c|}{ J095551.2 } & \multicolumn{4}{|c|}{ J095550.6 } \\
\hline & $N_{H}^{a}$ & $\bar{\Gamma}$ & $L_{X}{ }^{b}$ & $\chi^{2} / d o f$ & $N_{H}^{a}$ & $\bar{\Gamma}$ & $L_{X}{ }^{b}$ & $\chi^{2} / d o f$ & $N_{H}^{a}$ & $\bar{\Gamma}$ & $L_{X}{ }^{b}$ & $\overline{\chi^{2} / d o f}$ \\
\hline 1 & & & & & $21_{-2.3}^{+5.9}$ & $2.15_{-0.36}^{+0.95}$ & $17 \pm 0.7$ & $1.0 / 81$ & $2.86_{-0.42}^{+0.45}$ & $2.85_{-0.33}^{+0.36}$ & $2.4 \pm 0.1$ & $0.9 / 48$ \\
\hline 9 & $3.63_{-0.75}^{+0.75}$ & $1.74_{-0.51}^{+0.51}$ & $7.8 \pm 0.2$ & $0.8 / 69$ & $15_{-3.2}^{+9.3}$ & $1.07_{-0.61}^{+1.66}$ & $3.2 \pm 0.2$ & $0.9 / 17$ & $2.41_{-0.60}^{-0.42}$ & $2.81_{-0.52}^{+0.58}$ & $2.0 \pm 0.1$ & $1.1 / 20$ \\
\hline 10 & $3.19_{-0.17}^{+0.23}$ & $1.47_{-0.09}^{+0.12}$ & $12 \pm 0.1$ & $1.0 / 293$ & & & & & & & & \\
\hline 11 & $\begin{array}{r}-0.17 \\
3.56_{-0.17}^{+0.19}\end{array}$ & $\begin{array}{r}-0.09 \\
1.52_{-0.08}^{+0.09}\end{array}$ & $13 \pm 0.1$ & $1.1 / 331$ & $13_{-5.1}^{+2.6}$ & $0.66_{-0.48}^{+0.43}$ & $3.6 \pm 0.09$ & $1.4 / 70$ & $2.56_{-0.34}^{+0.35}$ & $2.72_{-0.28}^{+0.31}$ & $1.5 \pm 0.04$ & $1.5 / 58$ \\
\hline 12 & $3.34_{-0.34}^{+0.38}$ & $\begin{array}{l}1.27_{-0.18}^{+0.18} \\
\end{array}$ & $11 \pm 0.2$ & $0.9 / 126$ & $12_{-4.5}^{+10.5}$ & $0.61_{-0.52}^{+1.80}$ & $3.3 \pm 0.2$ & $1.2 / 16$ & $3.99_{-1.10}^{+1.30}$ & $4.46_{-0.12}^{+1.12}$ & $11.6 \pm 0.6$ & $1.7 / 12$ \\
\hline
\end{tabular}

NOTE.- All quoted uncertainties are $90 \%$ except for the luminosities which are $1 \sigma$.

The spectrum of J095551.0 in observation 9 suffered pile-up and a pile-up model was applied during spectral fit.

$a$ in units of $10^{22} \mathrm{~cm}^{-2}$

${ }^{b} 0.5-10 \mathrm{keV}$ unabsorbed luminosity in units of $10^{39} \mathrm{ergs} \mathrm{s}^{-1}$ (assuming $\mathrm{d}=3.6 \mathrm{Mpc}$ ).

atively hard spectra of these observations. To verify the contamination, we extracted combined spectra of all three sources using observations 11 and 12 for which the sources are well resolved. While the X-ray flux is dominated by the transient, the X-ray spectra become significantly harder with a photon index of $\sim 1$. This indicates that the hard spectra of the three contaminated observations are likely due to nearby sources. Furthermore, mild pile-up may also affect the spectra. We therefore do not include the spectral fits in Table 2. The best fitting power-law spectrum of observation 11 is shown in Figure 2 .

J095551.2 is the next brightest source near the transient. We first fitted the spectra with an absorbed power-law model and the spectral parameters are shown in Table 2. Three of the fits are acceptable and the spectra turn over at about $4 \mathrm{keV}$ suggesting very high absorption. The best fitted $N_{H}$ is about $(1-2) \times 10^{23} \mathrm{~cm}^{-2}$ which is an order of magnitude greater than the other two nearby sources. In addition, all spectra are very hard with $\Gamma \lesssim 1$ except for observation 1 . For observation 11, a soft excess is clearly seen in the spectrum (Fig. 2) and the fit is much poorer than the others. Indeed, soft excess is seen in all spectra but with larger uncertainties due to shorter exposure time or smaller collecting area of ACIS-I below 2 $\mathrm{keV}$. Soft excess is a common feature of AGN with an ionized absorber. We then refitted the spectrum (observation 11) with an additional ionized absorber (absori model in XSPEC; Zdziarski et al. 1995). The fit is acceptable with a reduced $\chi^{2}$ of 1.06, and the best-fit photon index steepens to 2 with a large absorbing column of $N_{H}=9 \times 10^{23} \mathrm{~cm}^{-2}$ (see Fig. 3); the absorbed $0.5-10 \mathrm{keV}$ flux is $10^{-12} \mathrm{ergs} \mathrm{cm}^{-2} \mathrm{~s}^{-1}$.

Apart from observation 11, the X-ray spectra of J095550.6 can be fitted with an absorbed power-law with $N_{H} \sim 3 \times 10^{22}$ $\mathrm{cm}^{-2}$ and a photon index of $>2.7$. Observation 11 has the longest exposure time and the X-ray spectrum of J095550.6 is clearly more complicated. It cannot be fitted with any single component model. Instead, a combination of RaymondSmith model and power-law model is required. In addition, emission lines are clearly seen in the X-ray spectrum (Fig. 4). The X-ray spectrum indicates that J095550.6 is either a nearby foreground star or a supernova remnant in M82. We will show in $\$ 4.3$ that J095550.6 is indeed a young supernova remnant in M82. 

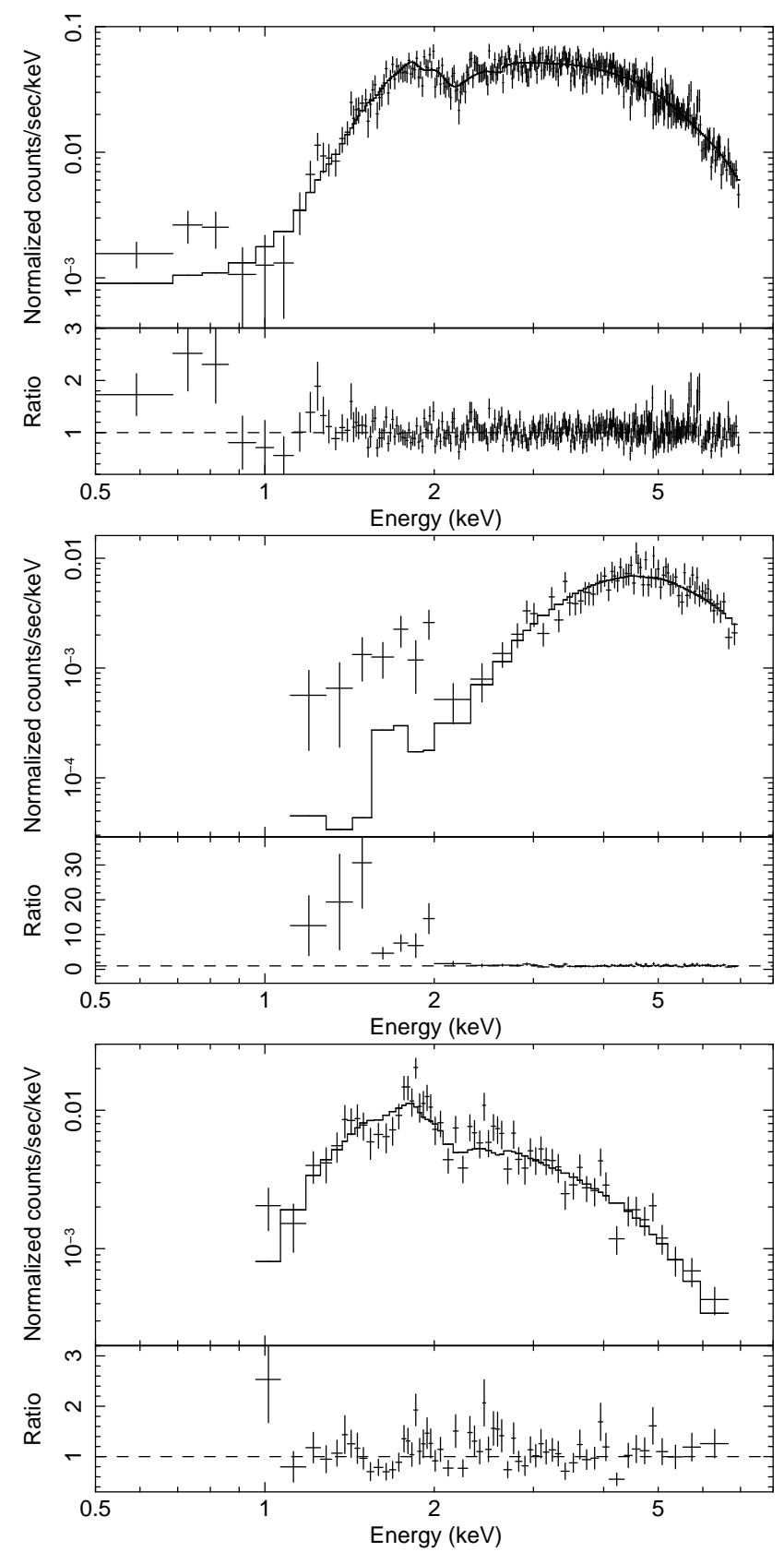

FIG. 2.- Power-law spectral fits of J095551.0 (top), J095551.2 (middle), and J095550.6 (bottom). Spectra are from observation 11. See Table 2 for spectral parameters.

\subsection{X-ray Variability}

With the spectral fits, we can estimate the X-ray fluxes of the three sources and study the long-term variability. We limit our analysis to Chandra data because the three sources as well as M82 X-1 are not resolved with Einstein, ROSAT, and XMM-Newton. The luminous X-ray source, J095551.0, displays strong variability on the timescales of months (see Fig. 5). In particular, the source was not detected in 1999 September and reappeared in 1999 October. It was below the detection limit again in 2000 January and then turned back on in 2000 March. Figure 5 shows the long-term X-ray lightcurve of J095551.0 from 1999 September to 2005 August. For the HRC-I observations and observations 4, 6, 7, and 8, we estimated the X-ray flux by assuming an absorbed power-law

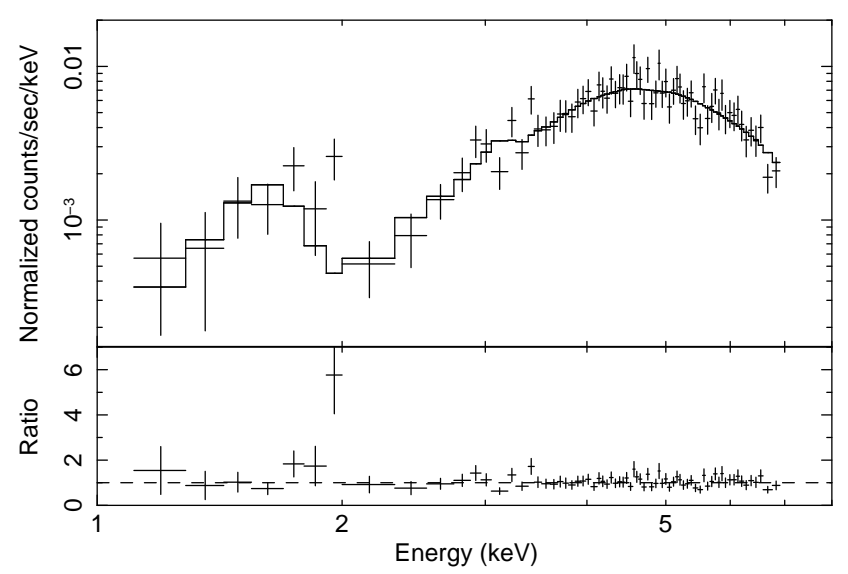

FIG. 3.- Chandra spectrum (from observation 11) of J095551.2 with an ionized absorber plus power-law model $\left(N_{H}=9 \times 10^{23} \mathrm{~cm}^{-2}, \Gamma=2\right.$, ionization parameter $\left.\xi=882, \chi^{2} / d o f=1.06 / 69\right)$. We fixed the absorber temperature and $\mathrm{Fe}$ abundance at $10^{5} \mathrm{~K}$ and solar values, respectively as the fit was not sensitive to these parameters.

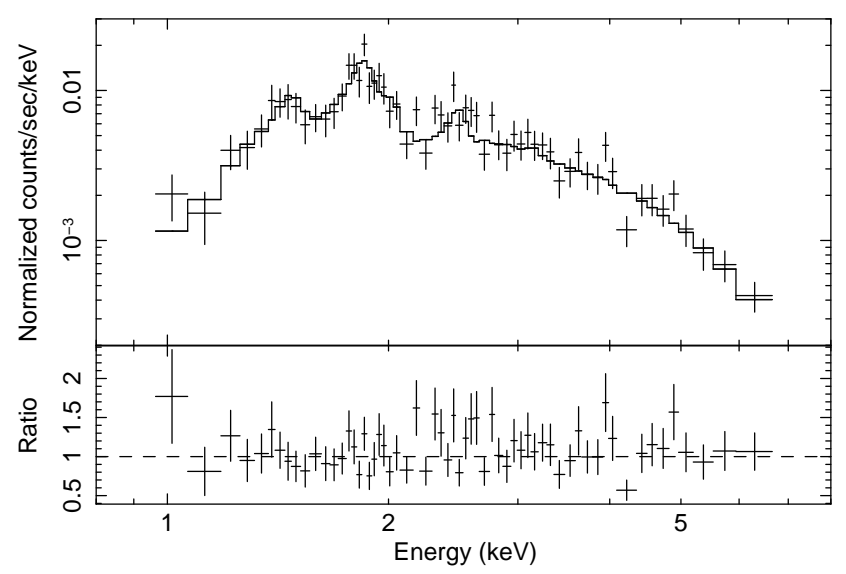

FIG. 4.- Chandra spectrum (from observation 11) of J095550.6 with an absorbed Raymond-Smith plus power-law model $\left(N_{H}=2.9 \times 10^{22} \mathrm{~cm}^{-2}\right.$, $k T_{R S}=0.9 \mathrm{keV}, \Gamma=2.01, \chi^{2} /$ dof $=1.1 / 56$ ).

model with $N_{H}=3 \times 10^{22} \mathrm{~cm}^{-2}$ and a photon index of 1.5. When the source is active, the X-ray luminosity shows very little variability at $(7-13) \times 10^{39} \mathrm{ergs} \mathrm{s}^{-1}$. We determined the $90 \%$ upper limit when the source was undetected; a nearby diffuse emission region was used as the background since the source is contaminated by strong diffuse emission. We note that Feng \& Kaaret (2007) reported a much lower upper limit (without statistical significance) by assuming the brightest pixel around the source region.

J095551.2 has a soft spectrum $(\Gamma=2)$ during the first observation and it becomes much harder $(\Gamma \lesssim 1)$ in subsequent observations. Because of the high $N_{H}$, the flux is very sensitive to the photon index. For instance, if we fit the spectra with an ionized absorber plus power-law model fixing the spectral parameters except for the normalization as in observation 11, the luminosities are $\sim 10^{40} \mathrm{ergs} \mathrm{s}^{-1}$. Hence, the source does not show significant variability.

For J095550.6, the apparent softening during the last observation is likely an artifact because of the low count rate. We used the same Raymond-Smith plus power-law model as in observation 11 and the spectrum can be fitted equally well. The resulting luminosity is about $2.4 \times 10^{39} \mathrm{ergs} \mathrm{s}^{-1}$, consistent with other observations. Therefore, the X-ray flux of J095550.6 is consistent with being constant. 


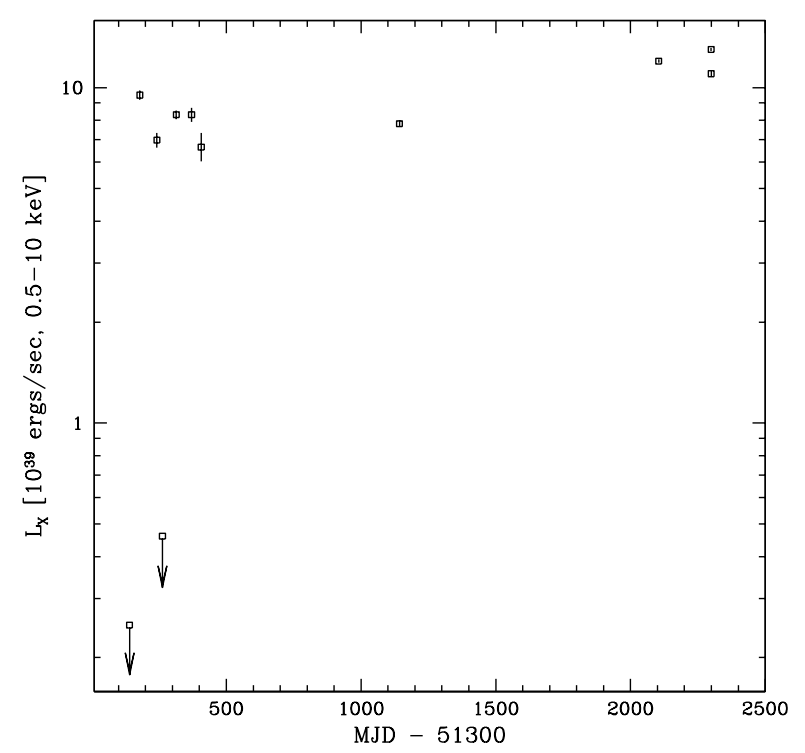

FIG. 5.- Long-term X-ray lightcurve of the ultraluminous X-ray transient, J095551.0. The luminosities are determined by spectral fits (see Table 2). For the HRC-I observation and observations $4,6,7$, and 8 , we assume an absorbed power-law spectral model with $N_{H}=3 \times 10^{22} \mathrm{~cm}^{-2}$ and $\Gamma=1.5$. For non-detections, 90\% upper limits are shown.

We also study the short-term variability of our targets. We extracted the source and background lightcurves from the 0.3$7 \mathrm{keV}$ event files except that there is no energy filter for HRCI data. We applied similar procedure as discussed in $\S 3.2$ to define the source and background regions of our targets. All three sources do not show significant variability on timescale of hours. We show the short-term lightcurves of the transient in Figure 6.

\subsection{Radial Profile}

We investigated the spatial extent of the ULXs using observation 11 for which the sources have the highest number of counts and are well resolved. Soft $(0.3-3 \mathrm{keV})$ and hard (3-7 keV) band counts were extracted from energy filtered images. We also modelled the point spread functions (PSF) of each source using Chandra Ray Tracer (ChaRT) and compared them with the measured radial profile of the three sources. Counts from these images were extracted in identical manner and normalized by the innermost nuclear annulus. The resulting radial profiles are shown in Figure 7.

The surface brightness distributions of all sources but J095551.2 in the hard and soft bands are very similar. They are centrally peaked and lie above background level out to radius $\approx 2^{\prime \prime}$ with the exception of J095551.2 for which the contamination from background is significant in the soft band. The radial profiles were compared with the PSF models; we do not find significant difference based on a KolmogorovSmirnov (K-S) test for all sources but J095551.2.

\subsection{Near IR Imaging}

In order to compare the Chandra and HST NICMOS images, we first aligned the two images. For the X-ray image, we used the HRC-I observation taken on 1999 October 28 (observation 3 ) as the reference frame because it has a wide fieldof-view and moderate exposure. Furthermore, all three targets were active during this observation. We used CIAO tool wavdetect to detect X-ray sources in the HRC-I image. We compared the X-ray source list with the 2MASS catalog, and looked for coincidence of bright and isolated stellar objects. We found one star (2MASS 09551494+6936143) that is $<1^{\prime \prime}$ from the corresponding HRC-I position. From the ACIS-I observation (observation 9), the X-ray colors of the X-ray emitting star indicate that it has a very soft $X$-ray spectrum (84\% of the source counts come from $<1 \mathrm{keV}$ with no counts above $2 \mathrm{keV}$ ), consistent with a very soft X-ray source (Di Stefano $\&$ Kong 2004). The X-ray radiation is therefore likely due to the coronal emission from a foreground star. The star has a $R$ magnitude of 10.1 (Monet et al. 2003). We calculated the X-ray to optical flux ratio as $\log \left(f_{X} / f_{R}\right)=\log f_{X}+5.67+0.4 R$ (Hornschemeier et al. 2001). With a count rate of $9.4 \times 10^{-4}$ $\mathrm{c} / \mathrm{s}$ in the ACIS-I detector and assuming a Raymond-Smith model with $k T_{R S}=0.3 \mathrm{keV}$ and $N_{H}=4 \times 10^{20} \mathrm{~cm}^{-2}$ (the Galactic value toward the direction of M82), the $0.3-10 \mathrm{keV}$ flux is $10^{-14} \mathrm{ergs} \mathrm{cm}^{-2} \mathrm{~s}^{-1}$ and the corresponding $f_{X} / f_{R}$ is $5.1 \times 10^{-5}$, consistent with a foreground star.

Based on the 2MASS counterpart, the boresight correction that needs to be applied to the X-ray source positions is $0.87 \pm 0.56$ arcsec in R.A. and $0.73 \pm 0.27$ arcsec in decl.; the uncertainties are a quadratic sum of the errors on the Xray and 2MASS positions. To study the IR environment of the X-ray sources, we plot on the NICMOS image (Figure 8 ) the corrected X-ray positions with error circles given by the quadratic sum of the positional uncertainty for the X-ray source $\left(0.032^{\prime \prime}\right.$ for the transient and $0.063^{\prime \prime}$ for the other two sources), the uncertainty in the optical astrometry (2MASS to ACS astrometry and ACS to NICMOS astrometry; $0.2^{\prime \prime}$ ), and the uncertainty in the X-ray boresight correction $\left(0.62^{\prime \prime}\right)$. In the figure, we also plot the locations of several known superstar clusters and M82 X-1. As expected, this area shows many star forming regions with the presence of super-star clusters. The three luminous X-ray sources as well as M82 X-1 are located near star clusters. In particular, a near IR source is at the center of the error circle of J95551.0. The source is marginally resolved in the F160W image with a half-light radius of $0.6 \mathrm{pc}$ by fitting with a King model (N. McCrady, private communication). At that size the cluster likely has a mass of $<10^{5} M_{\odot}$. J095551.2, however, does not seem to have any counterpart. While there is no obvious counterpart within the X-ray error circle of J095550.6, some unresolved IR emission is seen. Furthermore, a super-star cluster, MGG-8, is just outside the $1 \sigma \mathrm{X}$-ray error circle locating 1 arcsec from the source. It is also worth noting that M82 X-1 is located just outside the super-star cluster, MGG-11.

\section{DISCUSSION}

\subsection{J095551.0: An Ultraluminous X-ray Transient}

The most intriguing behavior of J095551.0 is the X-ray variability. The source varies from below the detection limit, $\sim 2.5 \times 10^{38} \mathrm{ergs} \mathrm{s}^{-1}$ in the $0.5-10 \mathrm{keV}$ band, to $\sim 10^{40} \mathrm{ergs}$ $\mathrm{s}^{-1}$ on timescales between observations of $\sim 2$ months. Furthermore, the source shows recurrent outbursts. Recurrent ultraluminous transients are not common in nearby galaxies. By comparing ROSAT and XMM-Newton observations, Winter et al. (2006) found that most of the ULXs are persistent sources with less than a factor of 3 in flux variation over the timescale from ROSAT to XMM-Newton. Nevertheless, ultraluminous X-ray transients have been found in NGC 3628 (Strickland et al. 2001), M74 (Soria \& Kong 2002), NGC 300 (Kong $\&$ Di Stefano 2003), NGC 253 (Bauer \& Pietsch 2005), and M101 (Kong et al. 2004; Kong \& Di Stefano 2005). Two 

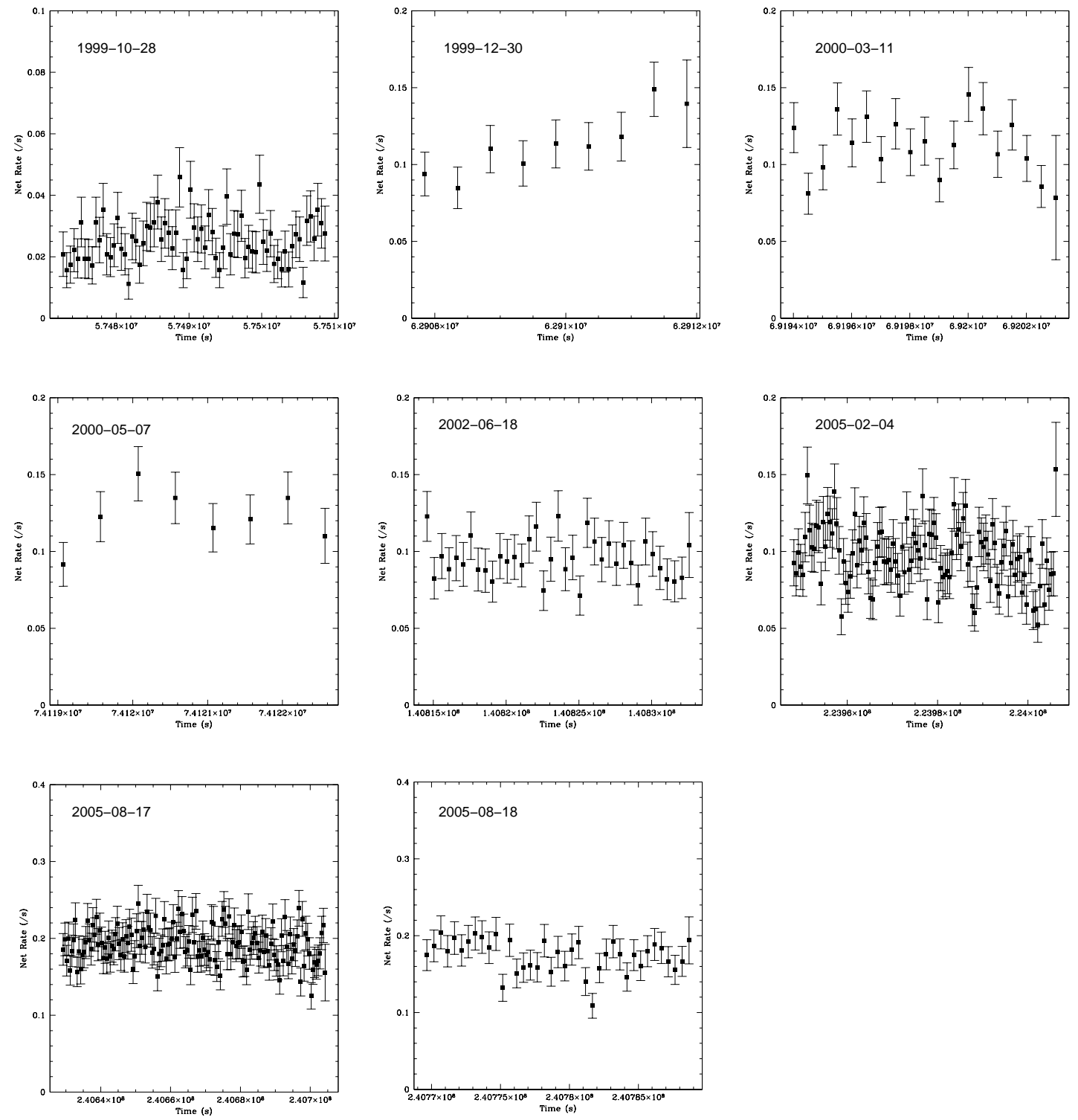

FIG. 6.- Chandra short-term lightcurves of the transient, J095551.0, when it is active. The time resolution of each plot is $500 \mathrm{~s}$. We do not show observation 8 due to its short exposure time $(1.2 \mathrm{ks})$. We note that the apparent difference in the count rate is due to different detectors and off-axis angle of the source.

of these sources (NGC 300 and M101) are ultraluminous supersoft sources with $k T \lesssim 0.1 \mathrm{keV}$. The ones in NGC 3628 and M74 are typical power-law sources with $\Gamma \sim 2$ while the ULX in NGC 253 can be described with a bremsstrahlung model with $k T=2.2 \mathrm{keV}$. J095551.0, however, has a much harder spectrum. These sources also show diverse luminosity range. The sources in NGC 253, M74, and NGC 300 only reach $L_{X} \gtrsim 10^{39} \mathrm{ergs} \mathrm{s}^{-1}$ suggesting that an IMBH is not necessary. On the other hand, the sources in NGC 3628 and M101 can have $0.3-8 \mathrm{keV}$ luminosities of $\sim 5 \times 10^{40} \mathrm{ergs} \mathrm{s}^{-1}$ with bolometric luminosities approaching $10^{41} \mathrm{ergs} \mathrm{s}^{-1}$. While we cannot totally rule out a stellar-mass black hole model, a black hole of intermediate mass is certainly an attractive scenario.

A factor of $>50$ in flux variation indicates that J095551.0 is a compact source while recurrent outbursts and hard Xray spectra can rule out the possibility that the source is a young supernova remnant. It is also unlikely to be a background AGN since AGN normally varies by a factor of $<10$ on timescales of days to months. However, transient AGNs are not unusual (e.g., Komossa et al. 2004; Grupe et al. 2004) and they belong to a class of AGNs called narrow-line Seyfert 1 galaxies. These galaxies have X-ray spectra much softer $(\Gamma>2.5$; e.g., Boller et al. 1996; Grupe et al. 2004) than J095551.0. The more likely scenario is that J095551.0 is a binary system with a black hole accretor. The high X-ray luminosity $\left(L_{X} \approx 10^{40} \mathrm{ergs} \mathrm{s}^{-1}\right)$ when it is active indicates that it may be an ULX with an IMBH. Assuming the emission is isotropic, the X-ray luminosity implies that the compact object is $\mathrm{a} \sim 100 M_{\odot}$ black hole. Many ULXs have a thermal component with a temperature of $\approx 0.1 \mathrm{keV}$ which is interpreted as evidence of IMBHs with masses of $\sim 100-1000 M_{\odot}$ (Miller et al. 2004). J095551.0, however, does not have any soft excess and the X-ray spectra are well fitted with an absorbed power-law model with photon index $\Gamma=1.3-1.7$. This resembles to the low/hard state of Galactic black hole X-ray binaries (McClintock \& Remillard 2006). It is therefore pos- 

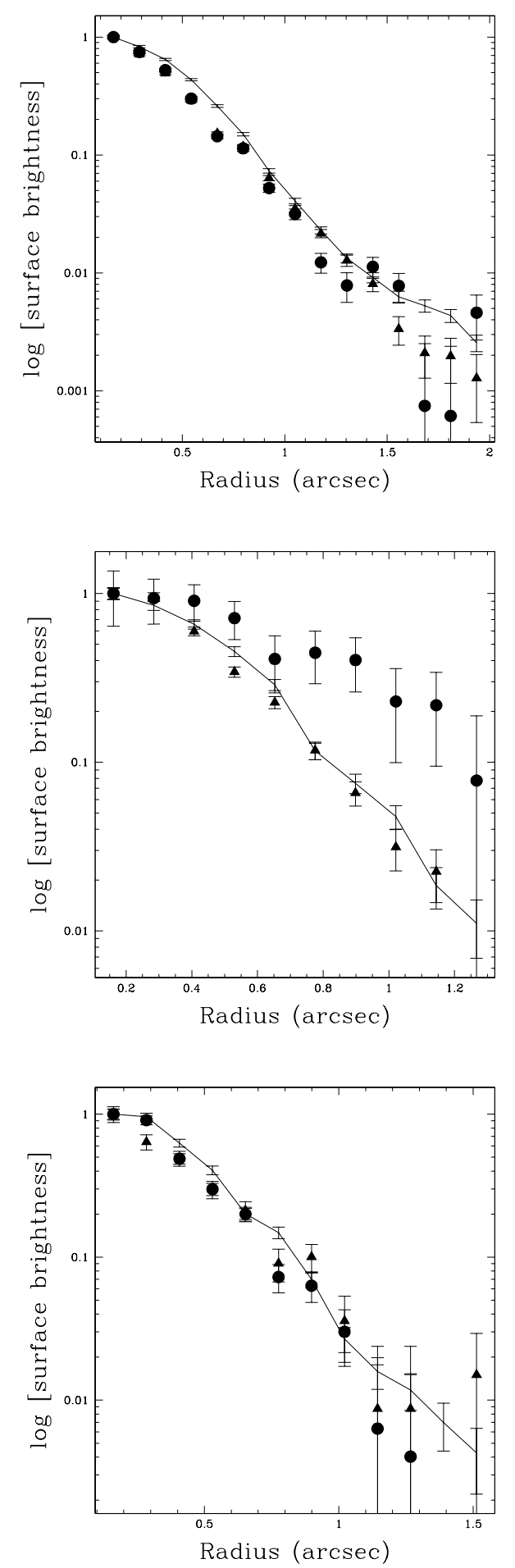

FIG. 7.- The soft (0.3-3 keV; solid points) and hard (3-7 keV; triangles) band radial profile of J095551.0 (top), J095551.2 (middle), and J095550.6 (bottom), compared to a Chandra PSF model (solid curve), from observation 11 .

sible that the source can be explained in the framework of the advection-dominated accretion flow (ADAF) model.

More recently, Yuan et al. (2007) apply an ADAF model to describe the X-ray emission of M82 X-1 and argue that the accreting compact object is an IMBH. During the low/hard state, the ADAF model predicts that the X-ray luminosity is about $<1 \%-10 \%$ of the Eddington luminosity. If J095551.0 is accreting at a rate similar to the hard-state of Galactic black hole X-ray binaries, this would imply a black hole mass of $\sim 1000-10^{4} M_{\odot}$. The estimate should be treated with caution because we assume that the X-ray spectra of the transient ULX are similar to that of Galactic black holes in the low/hard state. Indeed, pure power-law spectral model for ULXs is not uncommon; Chandra and XMM-Newton observations have revealed hard-state ULXs in several nearby galaxies (see e.g., Roberts et al. 2004; Winter et al. 2006). Hard-state ULXs may be good candidates to IMBHs. If the accreting object is instead a stellar-mass black hole in the hard state, the X-ray emission must be anisotropic in order to produce such a high $\mathrm{X}$-ray luminosity. However, the inner accretion disk of hardstate Galactic black hole X-ray binaries are truncated at large distances from the black hole and this may be a problem for the thick-disk plus central funnels anisotropic radiation model (King et al. 2001). Furthermore, the lack of short time variability of J095551.0 argues against the relativistic beaming model since this would require a very stable jet. It is worth noting that the observed photon index of J095551.0 is sometime harder than the typical hard-state value of $1.5<\Gamma<2.1$ for Galactic X-ray binaries (McClintock \& Remillard 2006). It is therefore not clear if we can directly compare with Galactic black hole X-ray binaries in the hard state. Alternatively, it may be a unique state that the ULX is a stellar-mass black hole accreting at very high rate.

In addition to the long-term timing variability, the X-ray spectra also vary. Excluding those observations taken with off-axis pointings, the photon index is consistent with 1.51.7 except for the last observation (see Table 2). In the last observation, the photon index becomes much harder with $\Gamma=1.27 \pm 0.18$. Moreover, the spectral change is quite dramatic. The observation taken one day earlier has a photon index of $1.52 \pm 0.10$ while the X-ray luminosity does not change significantly. On the other hand, the nearby source, J095551.2, does not show this dramatic change in the spectra. We also check the spectra of a few bright sources in the field for the last two observations; none of the sources displays this kind of spectral variability. We therefore can conclude that the spectral hardening is real. Such an X-ray spectrum is unusual for Galactic black hole X-ray binaries. The only exception is the fast X-ray nova, SAX J1819.3-2525 whose spectrum is extraordinary hard with $\Gamma=0.6-0.9$ during a flaring state (Markwardt et al. 1999; McClintock \& Remillard 2006). The last observation of J095551.0 is similar to SAX J1819.3-2525 but we note that the luminosity is indeed slightly lower than that measured one day earlier and the short-term light curve does not display strong variability.

Near IR and radio observations may provide additional clues about the nature of J095551.0. From the NICMOS image (Fig 8), a star cluster is at the center of the X-ray error circle of J095551.0, suggesting that the source is associated with the cluster. Indeed, young star clusters are ideal places to produce IMBHs via the collapse of very massive stars through runaway stellar collisions (Portegies Zwart et al. 2004). The coincidence of J095551.0 and a star cluster strongly suggests that the ULX is produced in the cluster and is consistent with a black hole of intermediate mass. Radio emission is also detected within the X-ray error circle (Körding et al. 2005; Kaaret et al. 2006). The radio source, known as 42.21+59.0, has been detected several times (e.g., McDonald et al. 2002). The flat spectrum (between 5 and $15 \mathrm{GHz}$ ) and extended size 


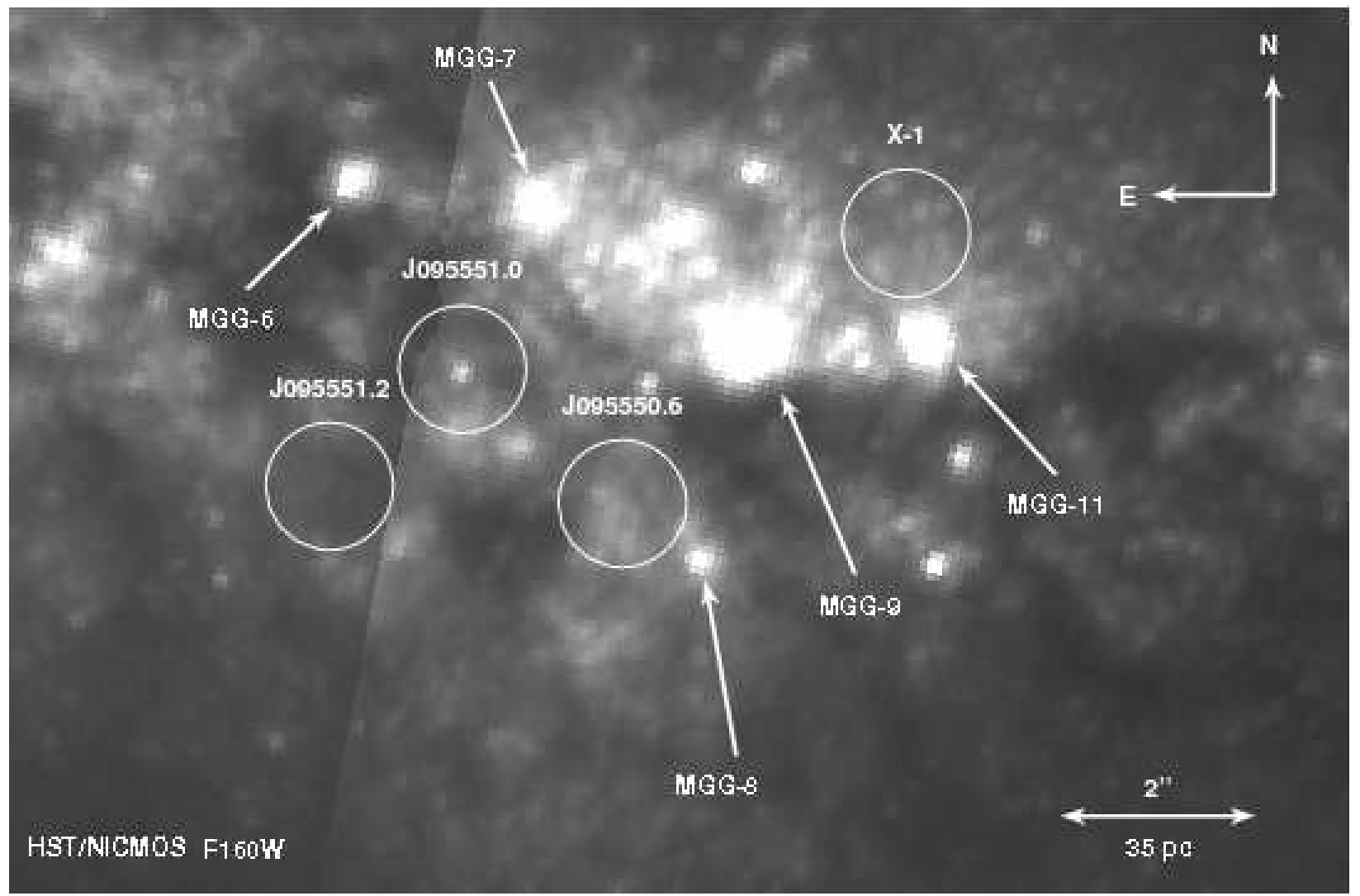

FIG. 8.- HST/NICMOS F160W image of the region around the X-ray sources analyzed in this paper (J095551.0, J095551.2, and J095550.6). The 1 $\sigma$ Chandra error circles $(0.66$ arcsec) are shown. We also label the locations of known super-star clusters and M82 X-1.

$(4.9 \mathrm{pc})$ suggest that it is a giant $\mathrm{H}$ II region with $94 \mathrm{O} 5$ stars (McDonald et al. 2002).

\subsection{J095551.2: A Highly Absorbed X-ray Source}

The X-ray spectrum of J095551.2 is very different compared to that of other nearby sources. The source has an unusually high $N_{H}\left(>10^{23} \mathrm{~cm}^{-2}\right)$ while the two nearby sources as well as M82 X-1 (Kaaret et al. 2006) have a $N_{H}$ of $\sim 3 \times 10^{22} \mathrm{~cm}^{-1}$, consistent with the extinction measured by IR observations. Furthermore, J095551.2 has a very flat spectrum $(\Gamma \lesssim 1)$ with soft excess below $2 \mathrm{keV}$. The high absorption column density may indicate that the source is a background AGN. AGN normally has a power-law spectral model with $\Gamma \sim 1.7-2$ (e.g., Page et al. 2006) while the spectrum of J095551.2 is much harder. A hard spectral index of AGN may be due to the presence of a reflection component and/or a complex absorber (Cappi et al. 2006). In $\$ 3.1$, we refitted the spectrum (observation 11) with an ionized absorber plus power-law model yielding an acceptable fit. The photon index steepens to 2 and the $0.5-10 \mathrm{keV}$ unabsorbed flux is significantly higher. The other two spectra (observations 9 and 12) can also be well fitted with an ionized absorbed plus power-law model with the spectral parameters fixed at the values determined in observation 11 except for the normalization. However, due to the low count rate of these two observations, it is not clear if the difference is real. On the other hand, the spectrum of the first observation might be different. The spectral parameters of observation 11 cannot fit the spectrum of the first observation. If we only fit all the spectra in the range of $2.5-7 \mathrm{keV}$ where soft excess is not crucial, the spectrum of the first observation is much softer $(\Gamma=2.9)$ than the others $(\Gamma \lesssim 1)$. Hence, the spectral change during the first observation might be real. Spectral change is also seen in some narrow-line Seyfert 1 galaxies such as NGC 4051 (Guainazzi et al. 1996; Ponti et al. 2006; see Leighly 1999 for a review). Therefore, J095551.2 is likely to be a highly absorbed background AGN. It is worth nothing that there is a H II region within the X-ray error circle $(42.56+580$; McDonald et al. 2002).

\subsection{J095550.6: A Young X-ray Supernova Remnant}

J095550.6 is unique because the X-ray spectrum cannot be fitted with simple spectral models. Instead, a combination of a Raymond-Smith model and a power-law model is required to describe the spectrum (see Fig. 4). Given the high $N_{H}$ that is consistent with the extinction of nearby region of M82, J095550.6 is unlikely to be a foreground star. The X-ray spectrum is not typical for X-ray binaries. We can also rule out a background AGN due to its unusual spectrum. The remaining possibility is that it is an X-ray luminous supernova remnant. In particular, it is evident that the spectrum (Fig. 4) is dominated by broad emission of $\mathrm{Mg}$ XII lines at $1.4 \mathrm{keV}$, Si K shell lines at $1.8 \mathrm{keV}$, and $\mathrm{S} \mathrm{K}$ shell lines at $2.5 \mathrm{keV}$. Strong emission lines are also seen in some luminous X-ray emitting supernova such as SN 1978K (Schlegel et al. 2004). Indeed, SN 1978K is also the first known supernovae with X-ray luminosity above $10^{39} \mathrm{ergs} \mathrm{s}^{-1}$. For direct comparison with SN 1978K, we refit the spectrum of J095550.6 with an absorbed MEKAL + power-law model. The spectral parameters are not sensitive to the model, with a best-fit temperature of $0.9 \mathrm{keV}$. This is slightly hotter than SN 1978K $(\sim 0.7 \mathrm{keV}$; Schlegel et al. 2004). The $0.5-10 \mathrm{keV}$ luminosity of J095550.6 is 
$2.2 \times 10^{39} \mathrm{ergs} \mathrm{s}^{-1}$.

Within the $\mathrm{X}$-ray error circle, there is a strong radio source known as 41.95+575 (McDonald et al. 2001,2002; Körding et al. 2005). $41.95+575$ is the brightest and most compact radio source in M82 and has been detected since 1965 (Muxlow et al. 2005). High resolution (3 mas) VLBI imaging shows that the source has a double-lobed structure (McDonald et al. 2001). The separation of the two brightest components is 22.4 mas in 2001 (Muxlow et al. 2005). Furthermore, multiple-epoch VLBI observations show that the separation is increasing at a rate of 0.24 mas $\mathrm{yr}^{-1}$. The radio flux also varied over the last 30 years. It has decreased in flux density at a rate of $\sim 8.8 \%$ per year. An age of around 100 years is estimated (Muxlow et al. 2005). The simplest explanation of the nature of J095550.6 is that it is a supernova event taking place within a high density molecular cloud (McDonald et al. 2001). We can also estimate the age of the remnant using the X-ray spectral fit. Following Kong et al. (2002), assuming J095550.6 is in the adiabatic expansion phase, the shock temperature can be written as $T_{s}=(0.18 \mathrm{keV})\left(R / t_{3}\right)^{2}$, where $R$ and $t_{3}$ are the radius (in units of parsecs) and age (in units of $1000 \mathrm{yr}$ ), respectively. Adopting a radius of 11 mas $(=0.19 \mathrm{pc})$, and $T_{s}=0.9 \mathrm{keV}$, we obtain $t \sim 100 \mathrm{yr}$ which is consistent with radio observations. It is worth noting that long-term radio observations have suggested that $41.95+575$ may be a radio afterglow of a 100 year old gamma-ray burst event (Muxlow et al. 2005).

\section{CONCLUSIONS}

We have used archival Chandra and HST/NICMOS data to study the physical nature of three ULXs near the center of M82. We found a recurrent transient ULX, J095551.0 from the Chandra data. During its active state, the X-ray luminosity is about $7 \times 10^{39}-1.3 \times 10^{40} \mathrm{ergs} \mathrm{s}^{-1}$ and it was turned off twice in 1999 and 2000 indicating a factor of $>50$ variability. This also rules out the possibility that it is a supernova remnant. The X-ray spectra can be fitted with a power- law model with photon index $\Gamma=1.3-1.7$ which is similar to Galactic black hole X-ray binaries in the low/hard state. We suggest that the X-ray emission might be explained in the framework of the ADAF model implying a black hole mass of $\sim 10^{3}-10^{4} M_{\odot}$. However, we cannot totally rule out that the source is in a unique spectral/luminosity state. In particular, spectral hardening was seen in one of the observations. We also examined near IR images taken with HST/NICMOS. We found a star cluster at the center of the X-ray error circle suggesting that the source is associated with the cluster.

With an unusually high column density $N_{H}>10^{23} \mathrm{~cm}^{-2}$ and a rather flat $\mathrm{X}$-ray spectrum $(\Gamma \lesssim 1)$ with an ionized absorber, it is suggested that the source J095551.2 is likely to be a background AGN. The source also shows spectral change similar to some narrow-line Seyfert 1 galaxies. In addition, there is a $\mathrm{H}$ II region known as $42.56+580$ associated with the X-ray source.

The source J095550.6 shows an unusual spectrum that cannot be fitted with a simple power-law model. Instead, the spectrum can be fitted with a Raymond-Smith model, accompanying with broad emissions of $\mathrm{Mg}$, $\mathrm{Si}$, and $\mathrm{S}$, indicating that it is a supernovae remnant with an age of $\sim 100$ years. Furthermore, a radio source known as $41.95+575$ is found associated with J095550.6 within the X-ray error circle. The long-term radio observations reveal that $41.95+575$ may be a radio afterglow of a 100-year old gamma-ray burst event.

Part of this work was carried out at the National Tsing Hua University, Taiwan, and we thank Hsiang-Kuang Chang for warm hospitality. We also thank Nate McCrady for providing the structural parameters of the star cluster associated with the transient ULX. The HST data presented in this paper were obtained from the Multimission Archive at the Space Telescope Science Institute (MAST). STScI is operated by the Association of Universities for Research in Astronomy, Inc., under NASA contract NAS5-26555.

Facilities: CXO (ACIS, HRC), HST (NICMOS, ACS/WFC)

\section{REFERENCES}

Alonso-Herrero, A., Rieke, G. H., Rieke, M. J., \& Kelly, D. M. 2003, AJ, 125,1210

Bauer, M., \& Pietsch, W. 2005, A\&A, 442, 925

Begelman, M. C. 2002, ApJ, 568, L97

Boller, T., Brandt, W. N., \& Fink, H. 1996, A\&A, 305, 53

Cappi, M., et al. 2006, A\&A, 446, 459

Colbert, E. J. M., \& Mushotzky, R. F. 1999, ApJ, 519, 89

Di Stefano, R., \& Kong, A. K. H. 2004, ApJ, 609, 710

Ebisawa, K., Życki, P., Kubota, A., Mizuno, T., \& Watarai, K. 2003, ApJ, 597,780

Feng, H, \& Kaaret, P. 2007, ApJ, submitted, arXiv:0706.3233

Foschini, L., et al. 2002, A\&A, 396, 787

Freedman, W. L., et al. 1994, ApJ, 427, 628

Grupe, D., Leighly, K. M., Burwitz, V., Predehl, P., \& Mathur, S. 2004, AJ, 128,1524

Guainazzi, M., Mihara, T., Otani, C., \& Matsuoka, M. 1996, PASJ, 48, 781

Hornschemeier, A. E., et al. 2001, ApJ, 554, 742

Kaaret, P., Alonso-Herrero, A., Gallagher, J. S., Fabbiano, G., Zezas, A., \& Rieke, M. J. 2004, MNRAS, 348, L28

Kaaret, P., Simet, M. G., \& Lang, C. C. 2006, ApJ, 646, 174

King, A. R., Davies, M. B., Ward, M. J., Fabbiano, G., \& Elvis, M. 2001, ApJ, 552, L109

Komossa, S., Halpern, J., Schartel, N., Hasinger, G., Santos-Lleo, M., \& Predehl, P. 2004, ApJ, 603, L17

Körding, E., Falcke, H., \& Markoff, S. 2002, A\&A, 382, L13

Kong, A. K. H., Garcia, M. R., Primini, F. A., \& Murray, S. S. 2002, ApJ, 580, L125

Kong, A. K. H., Di Stefano, R., \& Yuan, F. 2004, ApJ, 617, L49

Kong, A. K. H., \& Di Stefano, R. 2005, ApJ, 632, L107
Körding, E., Colbert, E., \& Falcke, H. 2005, A\&A, 436, 427

Leighly, K. M. 1999, ApJS, 125, 317

Makishima, K., et al. 2000, ApJ, 535, 632

Markwardt, C. B., Swank, J. H., \& Morgan, E. H. 1999, IAU Circ., 7257, 2

Masetti, N., Foschini, L., Ho, L. C., Dadina, M., Di Cocco, G., Malaguti, G., \& Palazzi, E. 2003, A\&A, 406, L27

Matsumoto, H., Tsuru, T. G., Koyama, K., Awaki, H., Canizares, C. R., Kawai, N., Matsushita, S., \& Kawabe, R. 2001, ApJ, 547, L25

McClintock, J. E., \& Remillard, R. A. 2006, Compact stellar X-ray sources, 157

McDonald, A. R., Muxlow, T. W. B., Wills, K. A., Pedlar, A., \& Beswick, R. J. 2002, MNRAS, 334, 912

McDonald, A. R., Muxlow, T. W. B., Pedlar, A., Garrett, M. A., Wills, K. A., Garrington, S. T., Diamond, P. J., \& Wilkinson, P. N. 2001, MNRAS, 322, 100

Miller, J. M., Fabian, A. C., \& Miller, M. C. 2004, ApJ, 614, L117

Monet, D. G., et al. 2003, AJ, 125, 984

Mutchler, M., et al. 2007, PASP, 119, 1

Muxlow, T. W. B., Pedlar, A., Beswick, R. J., Argo, M. K., O’Brien, T. J., Fenech, D., \& Trotman, W. 2005, Memorie della Societa Astronomica Italiana, 76,586

Page, M. J., et al. 2006, MNRAS, 369, 156

Ponti, G., Miniutti, G., Cappi, M., Maraschi, L., Fabian, A. C., \& Iwasawa, K. 2006, MNRAS, 368, 903

Portegies Zwart, S. F., Baumgardt, H., Hut, P., Makino, J., \& McMillan, S. L. W. 2004, Nature, 428, 724

Roberts, T. P., Warwick, R. S., Ward, M. J., \& Goad, M. R. 2004, MNRAS, 349,1193

Schlegel, E. M., Kong, A., Kaaret, P., Di Stefano, R., \& Murray, S. 2004, ApJ, 603, 644 
Soria, R., \& Kong, A. .K. H. 2002, ApJ, 572, L33

Strickland, D. K., Colbert, E. J. M., Heckman, T. M., Weaver, K. A., Dahlem, M., \& Stevens, I. R. 2001, ApJ, 560, 707

Winter, L. M., Mushotzky, R. F., \& Reynolds, C. S. 2006, ApJ, 649, 730
Yuan, F., Taam, R. E., Misra, R., Wu, X.-B., \& Xue, Y. 2007, ApJ, 658, 282

Zdziarski, A. A., Johnson, W. N., Done, C., Smith, D., \& McNaron-Brown, K. 1995, ApJ, 438, L63 